\title{
There and back again, or always there? The evolution of rice combined strategy for Fe uptake
}

\author{
Felipe K. Ricachenevsky ${ }^{*}$ and Raul A. Sperotto ${ }^{2}$ \\ ${ }^{1}$ Laboratório de Fisiologia Vegetal, Departamento de Botânica, Centro de Biotecnologia, Universidade Federal do Rio Grande do Sul, Porto Alegre, Brazil \\ 2 Programa de Pós-Graduação em Biotecnologia, Centro de Ciências Biológicas e da Saúde, Centro Universitário UNIVATES, Lajeado, Brazil \\ ${ }^{*}$ Correspondence: felipecruzalta@yahoo.com.br
}

Edited by:

Marta Wilton Vasconcelos, Universidade Católica Portuguesa, Portugal

Reviewed by:

Takanori Kobayashi, Ishikawa Prefectural University, Japan

Manuel González-Guerrero, Universidad Politécnica de Madrid, Spain

Keywords: Fe uptake, combined strategy, molecular evolution, phylogenetic analyses, iron homeostasis

\section{Fe UPTAKE MECHANISMS AND TRANSCRIPTIONAL CONTROL}

Iron $(\mathrm{Fe})$ is an essential micronutrient for almost all living organisms and represents one of the most versatile metals in biology, being involved in many ubiquitous metabolic processes such as respiration and photosynthesis, and required as a cofactor for numerous enzymes (Sperotto et al., 2010; Grillet et al., 2014a). In plants, Fe deficiency can cause severe chlorosis, growth arrest, and even plant death. Although highly abundant in the earth's crust, Fe phytoavailability is usually low, mainly because $\mathrm{Fe}^{3+}$ forms insoluble $\mathrm{Fe}$ oxides (Lemanceau et al., 2009). To circumvent this problem, plants developed mechanisms to acquire Fe from the rhizosphere (Sperotto et al., 2012; Grillet et al., 2014a).

Fe uptake mechanisms were classically separate into two strategies. Strategy I, or reduction strategy, is carried out by all plants except those from Poaceae family, and consists of: (a) $\mathrm{H}^{+}$extrusion by P-type ATPases to acidify the rhizosphere and increase $\mathrm{Fe}^{3+}$ solubility (e.g., AtAHA2, Arabidopsis $\mathrm{H}^{+}$-pump ATPase); (b) reduction of $\mathrm{Fe}^{3+}$ by a plasma membrane (PM)-bound ferric chelate reductase to more soluble $\mathrm{Fe}^{2+}$ (e.g., AtFRO2, Ferric Reductase Oxidase); and (c) $\mathrm{Fe}^{2+}$ absorption into root epidermal cells by transmembrane transporters (e.g., AtIRT1, Iron-Regulated Transporter) (Hindt and Guerinot, 2012; Ivanov et al., 2012). All three components of this strategy increase their activities during Fe deficiency. Strategy II, or chelation strategy, is used by plants from Poaceae family, and involves: (a) synthesis and release of small molecular weight compounds of the mugineic acid family called phytosiderophores (PS) into the rhizosphere, which bind $\mathrm{Fe}^{3+}$ with high affinity, via TOM1/OsZIFL4 (Nozoye et al., 2011; Ricachenevsky et al., 2011); and (b) $\mathrm{Fe}$ (III)-PS complex uptake into root cells by a Yellow Stripe/Yellow Stripe-Like (YSL) transporters. Both processes (PS excretion and $\mathrm{Fe}$ (III)-PS transport) are increased in response to Fe deficiency.

Since both maize (Zea mays) ys 1 mutant (defective for $\mathrm{Fe}$ (III)-PS transport) and Arabidopsis thaliana irt1 mutant (defective for $\mathrm{Fe}^{2+}$ transport) cannot survive under $\mathrm{Fe}$ deficiency conditions, it was first widely accepted that these two Fe uptake strategies were the main mechanisms for $\mathrm{Fe}$ acquisition in each plant group. However, later work on rice (Oryza sativa) showed that two functional $\mathrm{Fe}^{2+}$ transporters, OsIRT1 and OsIRT2, were expressed in roots upon Fe deficiency (Ishimaru et al., 2006; Walker and Connolly, 2008). It was proposed that rice uses a combined strategy, which has all features of a strategy II plant (PS release through TOM1/OsZIFL4 and Fe(III)-PS uptake through OsYSL15, the YS1 orthologInoue et al., 2009; Lee et al., 2009) and some features of a strategy I plant $\left(\mathrm{Fe}^{2+}\right.$ uptake using IRT transporters). The other two components of strategy I plants, proton extrusion, and $\mathrm{Fe}$ (III)-chelate reductase activity, were not detected in Fedeficient rice roots (Ishimaru et al., 2006). Further evidence for combined strategy was provided by rice plants carrying a mutation in the NICOTIANAMINE AMINOTRANSFERASE (NAAT) gene, a key enzyme in PS synthesis. This mutant, which lacks PS, is able to grow if $\mathrm{Fe}^{2+}$ is supplied as Fe source (Cheng et al., 2007). Based on these findings, it was proposed that the ability to absorb $\mathrm{Fe}^{2+}$ evolved in rice as an adaptation to the soil conditions in flooded paddies, where $\mathrm{Fe}^{2+}$ is more abundant than $\mathrm{Fe}^{3+}$ (Ishimaru et al., 2006; Walker and Connolly, 2008; Hindt and Guerinot, 2012). So far, rice is the only plant described to use the combined strategy mechanism.

A number of studies described key players and major transcriptional networks that control $\mathrm{Fe}$ homeostasis in both grasses and non-grasses (Hindt and Guerinot, 2012; Ivanov et al., 2012). Interestingly, orthologous genes have been described in rice and Arabidopsis thaliana, showing similar roles. The bHLH transcription factor FIT (FER-like irondeficiency-induced transcription factor) from $A$. thaliana interacts with bHLH038 and bHLH039 to regulate IRT1 and FRO2 under Fe deficiency (Yuan et al., 2008). FIT has no ortholog in rice, but bHLH38/39 are highly similar to OsIRO2 (Hindt and Guerinot, 2012), a known downstream regulator of $\mathrm{Fe}$ deficiencyresponsive genes. OsIRO2 regulates the $\mathrm{Fe}(\mathrm{III})-\mathrm{PS}$ transport-related genes, but not OsIRT1 (Ogo et al., 2007). OsIDEF1, acting upstream of OsIRO2, and OsIDEF2, are transcriptional regulators of distinct but partially overlapping branches of $\mathrm{Fe}$ deficiency response in rice (Ogo et al., 2008; Kobayashi et al., 2009) However, 
no ortholog for OsIDEF1 or OsIDEF2 was described in A. thaliana, although similar genes are found in the genomic sequence (Kobayashi and Nishizawa, 2012).

In A. thaliana, a second regulatory network is controlled by bHLH transcription factor named POPEYE (PYE), which targets distinct metal homeostasis genes. PYE seems to be regulated by interacting partners such as BRUTUS (BTS), an E3 ubiquitin ligase with metal and DNA binding domains that negatively regulates the response to $\mathrm{Fe}$ deficiency (Long et al., 2010; Kobayashi et al., 2013). In rice, Zheng et al. (2011) identified a negative regulator of the Fe deficiency response, OsIRO3, which could be the ortholog of PYE (Hindt and Guerinot, 2012). Interestingly, BTS orthologs OsHRZ1 and OsHRZ2 were recently characterized as negative regulators of $\mathrm{Fe}$ uptake and $\mathrm{Fe}$ utilization genes (Kobayashi et al., 2013). Thus, it seems that control of $\mathrm{Fe}$ deficiency response is partly conserved between A. thaliana and rice. Moreover, Urzica et al. (2012) performed a trans-system analysis looking for genes responsive to low Fe supply in Chlamydomonas reinhardtii, $A$. thaliana and rice, and observed that BTS/HRZs, IRT1, and IRT2 are conserved throughout the plant lineage.

\section{EVOLUTION OF THE Fe DEFICIENCY RESPONSE}

Currently there is no model for the evolution of Fe deficiency response in plants, especially in Poaceae, and few studies have focused on testing the hypothesis that rice is the only combined strategy species. Considering the available evidence, two models are possible (Figure 1A). In the first, named "recent combined strategy," combined strategy is an evolutionary novelty restricted to rice or close ancestral species, not shared with other extant species from Poaceae, which all use strategy II, and rice has acquired ability to induce an IRT1-like transporter under Fe deficiency, partially resembling strategy $\mathrm{I}$, as an adaptation to flooding. It implies that Poaceae last common ancestor (LCA) has lost strategy I-based Fe acquisition capacity and gained strategy II-based Fe uptake mechanism before diversification within the family (Figure 1A).

In the second model, named "ancient combined strategy," combined strategy is considered an evolutionary ancient trait (Figure 1A). Poaceae LCA has gained the ability to use strategy II for Fe uptake, but maintained strategy I. During Poaceae diversification and speciation, both strategy I and strategy II-specific genes were available for natural selection, and thus distinct groups could have adapted differently to respond to low $\mathrm{Fe}$ condition. As an example, the rice lineage has maintained IRT1-like up-regulation from the original strategy I response, while rhizosphere acidification and Fe reduction traits were lost. While all Poaceae would use the more efficient $\mathrm{Fe}$ acquisition mechanism strategy II (Curie and Briat, 2003), the model predicts that extant species could also show partial strategy I as rice does (i.e., IRT1-like $\mathrm{Fe}^{2+}$ uptake).

Indirect evidence favors the ancient combined strategy model. OsIRT1 ortholog in barley (Hordeum vulgare), HvIRT1, is also up-regulated by Fe deficiency and transports $\mathrm{Fe}, \mathrm{Mn}, \mathrm{Zn}$, and $\mathrm{Cd}$ (Pedas et al., 2008). The maize ortholog, ZmIRT1, was both described as not Fe regulated by Nozoye et al. (2013) and as Fe regulated by Li et al. (2013). Either way, Li et al. (2013) observed strong up-regulation of ZmIRT1 under Fe deficiency and showed ZmIRT1 ability to complement $\mathrm{Fe}$ and $\mathrm{Zn}$ uptake-defective yeast strains. Interestingly, IRT1-like genes described in Poaceae clustered together with AtIRT1 in a phylogenetic analysis (Li et al., 2013). AtIRT2 and OsIRT2, two genes similar to AtIRT1 and OsIRT1 that code for Fe transporters (Ishimaru et al., 2006; Vert et al., 2009), are part of the same cluster. Strikingly, AtIRT1/AtIRT2 and OsIRT1/OsIRT2 gene pairs are localized in tandem in their respective genomic regions and, despite monocot/dicot divergence dates 120 to 200 million years ago (Salse et al., 2002), they still show some degree of microsynteny (Figure 1B). These data indicate that OsIRT1 (and probably OsIRT2) shares not only functional similarity but also common evolutionary origin with AtIRT1, as the ancient combined strategy model predicts. Moreover, phenolics were described as important for Fe deficiency response in $A$. thaliana and rice, indicating that less understood aspects of Fe deficiency response are conserved between strategy I and strategy II plants (Bashir et al., 2011; Rodríguez-Celma et al., 2013; Fourcroy et al., 2014; Schmid et al., 2014).

We should also consider the likeliness of each model. In recent combined strategy model, the LCA IRT1 gene would have lost Fe deficiency responsiveness, presumably through deleterious mutations related to promoter activity (i.e., hampering transcription factor binding, interaction with enhancers, etc.). For OsIRT1 to be able to respond again to low Fe concentration in combined strategy, such mutations (or any changes that rendered IRT1 non-regulated) would need to be reversed. That implies re-activation of non-functional regulatory sequences on the promoter of the same gene, which is part of the ZIP gene family of around ten members in Poaceae genomes, several of them encoding $\mathrm{Fe}$ transporters (Li et al., 2013), and re-insertion into intricate regulatory circuits (Kobayashi and Nishizawa, 2012). Although possible, the ancient combined strategy model is more parsimonious, predicting that IRT1 function was conserved through plant lineage evolution, and that preference for combined strategy or strategy II in Poaceae was a late adaptation (Figure 1A).

\section{CONCLUSIONS AND FUTURE PERSPECTIVES}

It has long been established that Poaceae species rely on strategy II mechanism, while all other plant groups use strategy I. Rice has been considered an exception to the Poaceae-strategy II rule (Ishimaru et al., 2006; Sperotto et al., 2012); however, although it is clear that strategy II is the main mechanism for Fe uptake in graminaceous plants, the presence of IRT1-based $\mathrm{Fe}^{2+}$ transport in roots might play a non-overlapping role, not only in rice but in other species (Ishimaru et al., 2006; Pedas et al., 2008; Li et al., 2013). The ancient combined strategy model proposed here states that AtIRT1 ortolog kept their ancient function, observed early in the plant lineage (Urzica et al., 2012), all the way from monocot/eudicot split to extant Oryza 


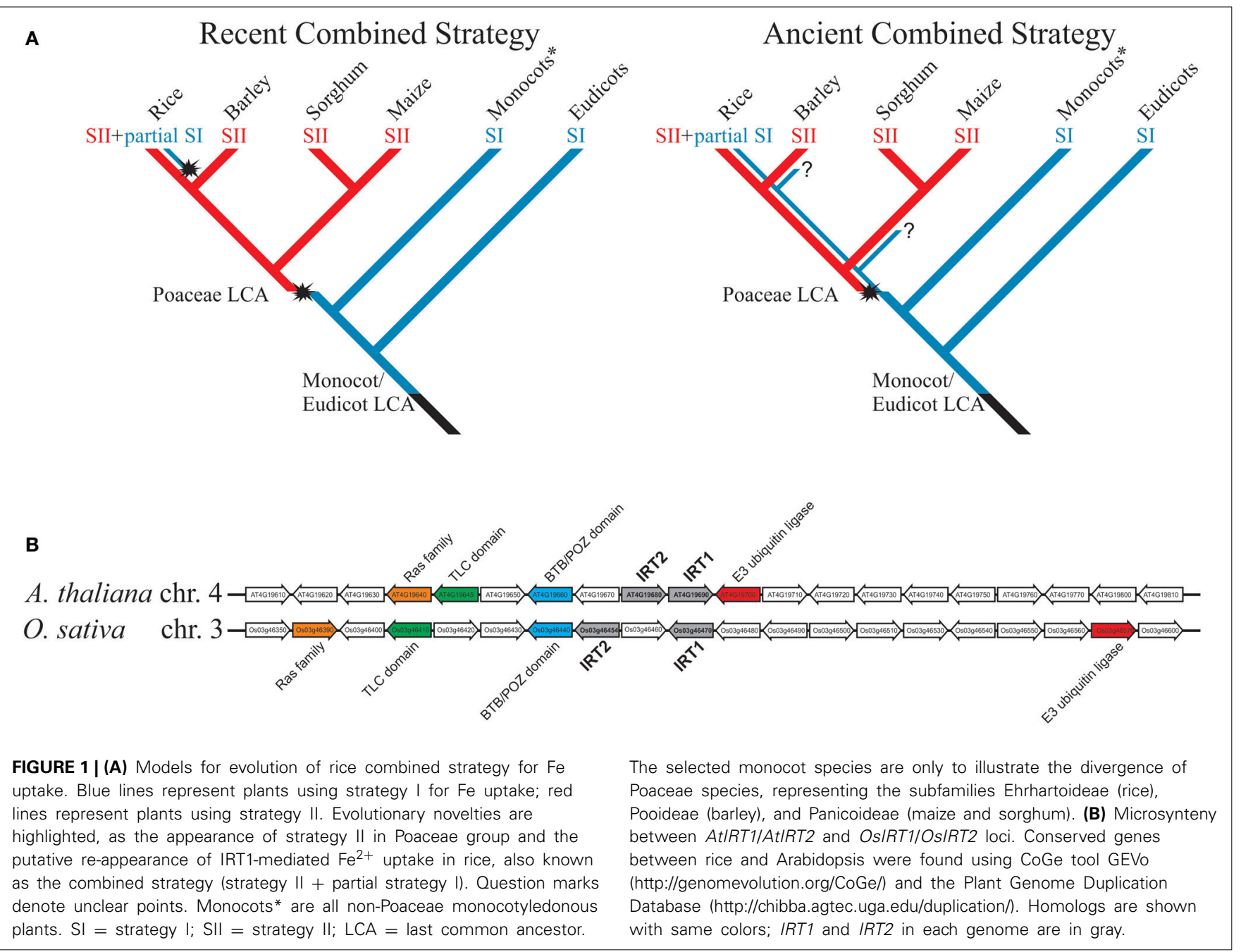

sativa species, rather than re-emerged with the same function in rice (Figure 1). Then, it is possible that distinct Poaceae subfamilies and species evolved independently to strategy II-exclusive or combined strategylike strategies.

Many studies have focused on Fe acquisition genes and underlying signaling pathway controlling these strategies, but most available data is on model species A. thaliana and rice (Hindt and Guerinot, 2012; Ivanov et al., 2012; Kobayashi and Nishizawa, 2012). Besides these, studies in maize, barley and Brachypodium distachyon have indicated the role of YSL transporters in Fe(III)-PS acquisition (Curie et al., 2001; Murata et al., 2006; Yordem et al., 2011). However, the role of IRT1-like transporters is still poorly understood. Studies on the role of IRT1, as well as other strategy I-related genes such as $\mathrm{Fe}^{3+}$-reductase, in Poaceae species, should shed light into how exclusive rice combined strategy is.

We should also consider that other species might use variants of strategy I and strategy II, or even rely on distinct mechanisms. Recently, it was demonstrated that peanut (Arachis hypogaea), an eudicot, is able to absorb $\mathrm{Fe}$ (III)PS complexes through AhYSL1 transporter. The complexes, however, are only present after intercropping with maize, which secretes PS in the soil, increasing Fe efficiency of peanut (Xiong et al., 2013). An exciting new study described a previously unknown Fe uptake mechanism, where $\mathrm{Fe}$ is delivered to embryos of pea (Pisum sativum) and A. thaliana in the form of $\mathrm{Fe}$ (III)-malate/citrate complexes, and is then chemically reduced to $\mathrm{Fe}^{2+}$ by ascorbate, which is effluxed from embryos, for subsequent uptake (Grillet et al., 2014b).

With lowering costs and increased access to technologies such as nextgeneration sequencing (Mardis, 2013) and genome editing (Gaj et al., 2013), it is becoming feasible to perform comparative genomics and transcriptomic studies with plants species for which genetic resources are not available, and eventually test key genes identified. These comparisons will allow testing the models discussed here, as well as uncovering new genes and strategies for $\mathrm{Fe}$ acquisition.

\section{ACKNOWLEDGMENTS}

We apologize to authors whose relevant work could not be cited due to the lack of space. The authors are supported by CNPq and Centro Universitário UNIVATES. 


\section{REFERENCES}

Bashir, K., Ishimaru, Y., Shimo, H., Kakei, Y., Senoura, T., Takahashi, R., et al. (2011). Rice phenolics efflux transporter 2 (PEZ2) plays an important role in solubilizing apoplasmic iron. Soil Sci. Plant Nutr. 57, 803-812. doi: 10.1080/00380768.2011. 637305

Cheng, L., Wang, F., Shou, H., Huang, F., Zheng, L., He, F., et al. (2007). Mutation in nicotianamine aminotransferase stimulated the $\mathrm{Fe}(\mathrm{II})$ acquisition system and led to iron accumulation in rice. Plant Physiol. 145, 1647-1657. doi: 10.1104/pp.107.107912

Curie, C., and Briat, J. F. (2003). Iron transport and signaling in plants. Ann. Rev. Plant Biol. 54, 183-206. doi: 10.1146/annurev.arplant. 54.031902 .135018

Curie, C., Panaviene, Z., Loulergue, C., Dellaporta, S. L., Briat, J. F., and Walker, E. L. (2001). Maize yellow stripe1 encodes a membrane protein directly involved in Fe(III) uptake. Nature 409, 346-349. doi: $10.1038 / 35053080$

Gaj, T., Gersbach, C. A., and Barbas, C. F. 3rd. (2013). ZFN, TALEN, and CRISPR/Cas-based methods for genome engineering. Trends Biotechnol. 31, 397-405. doi: 10.1016/j.tibtech.2013. 04.004

Fourcroy, P., Sisó-Terraza, P., Sudre, D., Savirón, M., Reyt, G., Gaymard, F., et al. (2014). Involvement of the ABCG37 transporter in secretion of scopoletin and derivatives by Arabidopsis roots in response to iron deficiency. New Phytol. 201, 155-167. doi: 10.1111/nph.12471

Grillet, L., Mari, S., and Schmidt, W. (2014a). Iron in seeds - loading pathways and subcellular localization. Front. Plant Sci. 4:535. doi: 10.3389/fpls.2013. 00535

Grillet, L., Ouerdane, L., Flis, P., Hoang, M. T., Isaure, M. P., Lobinski, R., et al. (2014b). Ascorbate efflux as a new strategy for iron reduction and transport in plants. J. Biol. Chem. 289, 2515-2525. doi: 10.1074/jbc.M113.514828

Hindt, M. N., and Guerinot, M. L. (2012). Getting a sense for signals: regulation of the plant iron deficiency response. Biochim. Biophys. Acta 1823, 1521-1530. doi: 10.1016/j.bbamcr.2012. 03.010

Inoue, H., Kobayashi, T., Nozoye, T., Takahashi, M., Kakei, Y., Suzuki, K., et al. (2009). Rice OsYSL15 is an iron-regulated iron(III)-deoxymugineic acid transporter expressed in the roots and is essential for iron uptake in early growth of the seedlings. J. Biol. Chem. 284, 3470-3479. doi: 10.1074/jbc.M806042200

Ishimaru, Y., Suzuki, M., Tsukamoto, T., Suzuki, K., Nakazono, M., Kobayashi, T., et al. (2006). Rice plants take up iron as an $\mathrm{Fe}^{3+}$. phytosiderophore and as $\mathrm{Fe}^{2+}$. Plant J. 45, 335-346. doi: 10.1111/j.1365-313X.2005.02624.x

Ivanov, R., Brumbarova, T., and Bauer, P. (2012). Fitting into the harsh reality: regulation of iron deficiency responses in dicotyledonous plants. Mol. Plant 5, 27-42. doi: $10.1093 / \mathrm{mp} /$ ssr065

Kobayashi, T., Itai, R. N., Ogo, Y., Kakei, Y., Nakanishi, H., Takahashi, M., et al. (2009). The rice transcription factor IDEF1 is essential for the early response to iron deficiency, and induces vegetative expression of late embryogenesis abundant genes. Plant J. 60, 948-961. doi: 10.1111/j.1365313X.2009.04015.x

Kobayashi, T., Nagasaka, S., Senoura, T., Itai, R. N., Nakanishi, H., and Nishizawa, N. K. (2013). Iron-binding haemerythrin RING ubiquitin ligases regulate plant iron responses and accumulation. Nat. Commun. 4, 2792. doi: 10.1038/ ncomms3792

Kobayashi, T., and Nishizawa, N. K. (2012). Iron uptake, translocation, and regulation in higher plants. Annu. Rev. Plant Biol. 63, 131-152. doi: 10.1146/annurev-arplant-042811105522

Lee, S., Chiecko, J. C., Kim, S. A., Walker, E. L., Lee, Y., Guerinot, M. L., et al. (2009). Disruption of OsYSL15 leads to iron inefficiency in rice plants. Plant Physiol. 150, 786-800. doi: 10.1104/pp.109.135418

Lemanceau, P., Bauer, P., Kraemer, S., and Briat, J. F. (2009). Iron dynamics in the rhizosphere as a case study for analyzing interactions between soils, plants and microbes. Plant Soil 321, 513-535. doi: 10.1007/s11104-009-0039-5

Li, S., Zhou, X., Huang, Y., Zhu, L., Zhang, S. Zhao, Y., et al. (2013). Identification and characterization of the zinc-regulated transporters, iron-regulated transporter-like protein (ZIP) gene family in maize. BMC Plant Biol. 13:114. doi: 10.1186/1471-2229-13-114

Long, T. A., Tsukagoshi, H., Busch, W., Lahner, B., Salt, D. E., and Benfey, P. N. (2010). The bHLH transcription factor POPEYE regulates response to iron deficiency in Arabidopsis roots. Plant Cell 22, 2219-2236. doi: 10.1105/tpc.110.074096

Mardis, E. R. (2013). Next-generation sequencing platforms. Annu. Rev. Anal. Chem. 6 , 287-303. doi: 10.1146/annurev-anchem-062012092628

Murata, Y., Ma, J. F., Yamaji, N., Ueno, D., Nomoto, K., and Iwashita, T. (2006). A specific transporter for iron(III)-phytosiderophore in barley roots. Plant J. 46, 563-572. doi: 10.1111/j.1365313X.2006.02714.x

Nozoye, T., Nagasaka, S., Kobayashi, T., Takahashi, M., Sato, Y., Uozumi, N., et al. (2011). Phytosiderophore efflux transporters are crucial for iron acquisition in graminaceous plants. J. Biol. Chem. 286, 5446-5454. doi: 10.1074/jbc. M110.180026

Nozoye, T., Nakanishi, H., and Nishizawa, N. K. (2013). Characterizing the crucial components of iron homeostasis in the maize mutants $y s 1$ and ys3. PLoS ONE 8:e62567. doi: 10.1371/journal.pone.0062567

Ogo, Y., Itai, R. N., Nakanishi, H., Kobayashi, T., Takahashi, M., Mori, S., et al. (2007). The rice bHLH protein OsIRO2 is an essential regulator of the genes involved in $\mathrm{Fe}$ uptake under $\mathrm{Fe}$ deficient conditions. Plant J. 51, 366-377. doi: 10.1111/j.1365-313X.2007.03149.x

Ogo, Y., Kobayashi, T., Nakanishi, I. R., Nakanishi, H., Kakei, Y., Takahashi, M., et al. (2008). A novel NAC transcription factor, IDEF2, that recognizes the iron deficiency-responsive element 2 regulates the genes involved in iron homeostasis in plants. J. Biol. Chem. 283, 13407-13417. doi: 10.1074/jbc.M708732200

Pedas, P., Ytting, C. K., Fuglsang, A. T., Jahn, T. P., Schjoerring, J. K., and Husted, S. (2008).
Manganese efficiency in barley: identification and characterization of the metal ion transporter HvIRT1. Plant Physiol. 148, 455-466. doi: 10.1104/pp.108.118851

Ricachenevsky, F. K., Sperotto, R. A., Menguer, P. K., Sperb, E. R., Lopes, K. L., and Fett, J. P. (2011). ZINC-INDUCED FACILITATORLIKE family in plants: lineage-specific expansion in monocotyledons and conserved genomic and expression features among rice (Oryza sativa) paralogs. BMC Plant Biol. 11:20. doi: 10.1186/1471-22 29-11-20

Rodríguez-Celma, J., Lin, W. D., Fu, G. M., Abadía, J., López-Millán, A. F., and Schmidt, W. (2013) Mutually exclusive alterations in secondary metabolism are critical for the uptake of insoluble iron compounds by Arabidopsis and Medicago truncatula. Plant Physiol. 162, 1473-1485. doi: 10.1104/pp.113.220426

Salse, J., Piégu, B., Cooke, R., and Delseny, M. (2002). Synteny between Arabidopsis thaliana and rice at the genome level: a tool to identify conservation in the ongoing rice genome sequencing project. Nucleic Acids Res. 30, 2316-2328. doi: 10.1093/nar/30.11.2316

Schmid, N. B., Giehl, R. F., Döll, S., Mock, H. P., Strehmel, N., Scheel, D., et al. (2014). FeruloylCoA 6'-Hydroxylase1-dependent coumarins mediate iron acquisition from alkaline substrates in arabidopsis. Plant Physiol. 164, 160-172. doi: 10.1104/pp.113.228544

Sperotto, R. A., Ricachenevsky, F. K., Stein, R. J., Waldow, V. A., and Fett, J. P. (2010). Iron stress in plants: dealing with deprivation and overload. Plant Stress 4, 57-69.

Sperotto, R. A., Ricachenevsky, F. K., Waldow, V. A., and Fett, J. P. (2012). Iron biofortification in rice: it's a long way to the top. Plant Sci. 190, 24-39. doi: 10.1016/j.plantsci.2012.03.004

Urzica, E. I., Casero, D., Yamasaki, H., Hsieh, S. I., Adler, L. N., Karpowicz, S. J., et al. (2012). Systems and trans-system level analysis identifies conserved iron deficiency responses in the plant lineage. Plant Cell 24, 3921-3948. doi: 10.1105/tpc.112. 102491

Vert, G., Barberon, M., Zelazny, E., Séguéla, M., Briat, J. F., and Curie, C. (2009). Arabidopsis IRT2 cooperates with the high-affinity iron uptake system to maintain iron homeostasis in root epidermal cells. Planta 229, 1171-1179. doi: 10.1007/s00425-0090904-8

Walker, E. L., and Connolly, E. L. (2008). Time to pump iron: iron-deficiency-signaling mechanisms of higher plants. Curr. Opin. Plant Biol. 11, 530-535. doi: 10.1016/j.pbi.2008.06.013

Xiong, H., Kakei, Y., Kobayashi, T., Guo, X., Nakazono, M., Takahashi, H., et al. (2013). Molecular evidence for phytosiderophore-induced improvement of iron nutrition of peanut intercropped with maize in calcareous soil. Plant Cell Environ. 36, 1888-1902. doi: 10.1111/pce. 12097

Yordem, B. K., Conte, S. S., Ma, J. F., Yokosho, K., Vasques, K. A., Gopalsamy, S. N., et al. (2011). Brachypodium distachyon as a new model system for understanding iron homeostasis in grasses: phylogenetic and expression analysis of Yellow Stripe-Like (YSL) transporters. Ann. Bot. 108, 821-833. doi: 10.1093/aob/mcr200 
Yuan, Y., Wu, H., Wang, N., Li, J., Zhao, W., $\mathrm{Du}$, J., et al. (2008). FIT interacts with AtbHLH38 and AtbHLH39 in regulating iron uptake gene expression for iron homeostasis in Arabidopsis. Cell Res. 18, 385-397. doi: 10.1038/cr. 2008.26

Zheng, L., Ying, Y., Wang, L., Wang, F., Whelan, J., and Shou, H. (2011). Identification of a novel iron regulated basic helix-loop-helix protein involved in $\mathrm{Fe}$ homeostasis in Oryza sativa. BMC Plant Biol. 10:166. doi: 10.1186/1471-2229$10-166$
Conflict of Interest Statement: The authors declare that the research was conducted in the absence of any commercial or financial relationships that could be construed as a potential conflict of interest.

Received: 24 February 2014; paper pending published: 04 April 2014; accepted: 22 April 2014; published online: 14 May 2014.

Citation: Ricachenevsky FK and Sperotto RA (2014) There and back again, or always there? The evolution of rice combined strategy for Fe uptake. Front. Plant Sci. 5:189. doi: 10.3389/fpls.2014.00189
This article was submitted to Plant Nutrition, a section of the journal Frontiers in Plant Science.

Copyright (ㄷ) 2014 Ricachenevsky and Sperotto. This is an open-access article distributed under the terms of the Creative Commons Attribution License (CC BY). The use, distribution or reproduction in other forums is permitted, provided the original author(s) or licensor are credited and that the original publication in this journal is cited, in accordance with accepted academic practice. No use, distribution or reproduction is permitted which does not comply with these terms. 